\title{
Los Sistemas de Previsión Social ante el IMPUESTO SOBRE LA RENTA DE LAS PERSONAS FÍsICAS
}

\author{
José Manuel Pérez Lara \\ Universidad de Granada
}

Se analiza el tratamiento que la Ley 40/1998, de 30 de diciembre, del Impuesto sobre la Renta de las Personas Físicas otorga, por un lado, a las aportaciones efectuadas para la financiación de los distintos sistemas de previsión social, Seguridad Social, Mutualidades de Funcionarios, Planes de Pensiones, Mutualidades de Previsión Social y Entidades Aseguradoras y. por otro, a las prestaciones que se derivan de los mismos, concedidas a los beneficiarios cuando se producen algunas de las contingencias protegidas, jubilación, invalidez o fallecimiento, haciendo una especial mención a las fórmulas de previsión social que instrumentan los compromisos por pensiones de las empresas con los trabajadores.

In this article we analyse how the Law on Personal Income Tax $140 / 1998$, 30th December) specifies, on the one hand, the contributions given to finance the different Social Service systems such as Social Security, National Health Care for Civil Servants, Pensions Plans, National Health Care and Insurance Agencies and, on the other hand, the corresponding benefits, given to the beneficiaries when some of the insured contingencies retirement, disability or death occur. Therefore we refer in particular to the Social Service arrangements by which the entrepreneurs commit themselves to procure pensions for their workers.

Palabras clave: IRPF, rendimientos del trabajo, Previsión Social. Planes de Pensiones. Mutualidades Key words: Personal Income Tax. Personal Eamed Income. Social Service

\section{INTRODUCCIÓN}

El artículo 41 de la Constitución Española establece que "los poderes públicos mantendrän un régimen público de Seguridad Social para todos los ciudadanos que garantice la asistencia y prestaciones sociales suficientes ante situaciones de necesidad, especialmente en el caso de desempleo. La asistencia y prestaciones complementarias serán libresn, y su artículo 50 que: "los poderes públicos garan- 
tizarán, mediante pensiones adecuadas y periódicamente actualizadas, la suff: ciencia económica a los ciudadanos durante la tercera edad..... En consecuen-: cia, se desprende la obligatoria existencia de un sistema público de pensiones: por mandato constitucional, y la posibilidad de la creación de sistemas privados de pensiones que complemente al público. Normalmente se ha aceptado la existencia de tres modelos para poder llevar a cabo la financiación de los sistemas: de previsión social. Por un lado, nos encontramos con un primer nivel, de carác ter estatal y generalizado, que cubre la pensiones asistenciales básicas, siendo un sistema no contributivo, en el sentido, de que el ciudadano no paga ninguna cantidad específicamente destinada a la cobertura de su pensión. Un segundo nivel, el profesional, siendo un sistema contributivo, ya que la pensión que se recibe guarda relación con las cantidades pagadas para poder tener derecho a la misma, y que, normalmente, es cofinanciado por el Estado, las empresas y los trabajadores, basado en el sistema de reparto. Este sistema de reparto, que es el adoptado por la Seguridad Social española, (art.87.1 del Real Decreto-Legislativo. 1/1994, por el que se aprueba el Texto Refundido de la Ley General de la Seguridad Social, dispone que: "El sistema financiero de todos los Regímenes que integran el sistema de la Seguridad Social será el de reparto, para todas las contingencias $y$ situaciones amparadas por cada uno de ellos....) consiste básicamente en que los pagos que va efectuando la población activa sirven para financiar las pensiones de la población jubilada existente en cada momento. Finalmente, un tercer nivel, de carácter complementario a los anteriores, voluntario y privado, que se financia a través de las aportaciones exclusivas efectuadas por los empresarios y/o trabajadores, basado en un sistema de capitalización o acumulación, es decir, cada sujeto va realizando aportaciones -normalmente a lo largo de su vida laboral- para la financiación de su propia pensión, en el sentido de que una entidad gestora invierta las aportaciones acumuladas de distintos sujetos para obtener una rentabilidad que junto con las aportaciones efectuadas permitan el pago de la futura pensión a cada sujeto.

Con la conjunción de todos los sistemas anteriores se cumplirá el mandato constitucional y sobre todo se conseguirá una estabilidad y paz social deseable por lo que se hace necesario utilizar todas las posibilidades políticas, jurídicas y económicas para la consecución de dichos fines. De las distintas posibilidades, el tratamiento fiscal que se conceda a los distintos sistemas de previsión, se convierte en un instrumento muy eficaz para el éxito o fracaso de los mismos. Es decir, el sistema tributario, o lo que es los mismo los tributos, no solámente sirven para recaudar dinero para de esta forma los poderes püblicos poder hacer frente a los gastos que le conllevan el desarrollo de las competencias concedidas por el ordenamiento, sino que también puede ser utilizados con una funétion 
extrafiscal, como preceptúa el artículo 4 de la Ley General Tributaria: "Los tributos; además de ser medios para recaudar ingresos puiblicos, ban de servir como instrumentos de la política económica general, atender a las exigencias de estabilidad y progreso sociales y procurar una mejor distribución de la renta nacional. Pues bien, el sistema impositivo espanool ha adoptado una serie de medidas para favorecer la instauración y desarrollo de distintos sistema de previsión social que pretende que las personas físicas tengan garantizadas unas prestaciones económicas en las situaciones de jubilación, invalidez o fallecimiento, parecidas en la cuantía a los salarios percibidos durante la vida laboral activa. Así, junto con el sistema público de previsión social -Seguridad Social y Mutualidades de Funcionarios-, se han ido potenciando en los últimos años los sistemas de privados de previsión social, básicamente los Planes de Pensiones y las Mutualidades de Previsión Social, relegando a las Entidades Aseguradoras. La evolución legislativa de los últimos años ha ido encaminada a clarificar y dar una ordenación homogénea a las diversas formulas empresariales de previsión social de los trabajadores, como se desprende de las distintas redacciones de la Disposición Adicional primera de la Ley 8/187, de 8 de junio, de Regulación de los Planes y Fondos de Pensiones ${ }^{1}$, que hasta el momento, ha concluido con la publicación del Real Decreto 1588/1999, de 15 de octubre, por el que se aprueba el Reglamento sobre instrumentación de los compromisos por pensiones de las empresas con los trabajadores y beneficiarios.

1. Disposición Adicional Primera: Protección de los compromisos con los trabajadores, modificada por la disposición adicional decimonovena de la Ley 30/1995, de 8 de noviembre. de Ordenación y Supervisión de los Seguros Privados, y por la Ley 6671997. de 30 de diciembre, de Medidas fiscales, administrativas y del orden social. Modificación incluía además las siguientes disposiciones transitorias como consecuencia de dicha modificacion:

- Disposición transitoria decimocuarta.. Régimen de los compromisos por pensiones ya asumidos. Modificada por la Disposición adicional decimotercera de la Ley 50/1998, de Medidas fiscales, administrativas y del orden social

- Disposición transitoria decimoquinta. Régimen transitorio de acomodación de los compromisos por pensiones mediante planes de pensiones. Modificada por la Ley 66/1997, de 30 de diciembre, de Medidas fiscales, administrativas y del orden social, por la disposición adicional undécima de la Ley 4071998, de 8 de diciembre, del Impuesto sobre la Renta de las Personas Físicas y. por la Disposición adicional decimotercera de la Ley 50/1998, de 30 de diciembre, de Medidas fiscales, administrativas y del orden social.

- Disposición transitoria decimosexta. Régimen fiscal transitorio de acomodación de los compromisos por pensiones. Modificada por la Ley 66/1997, de 30 de diciembre, de Medidas fiscales, administrativas y del orden social 
Siendo éste el objeto de este trabajo, especialmente, el tratamientø que la nuevai Ley del Impuesto sobre la Renta de las Personas Físicas concede, por un lado a las aportaciones efectuadas para la financiación de los sistema de previsión social $y$, por otro, a las prestaciones que se derivan de los mismos concedidas a los bene ficiarios cuando se producen alguna de las contingencias protegidas por estos sis:temas, haciendo una especial mención a la fórmulas de previsión social que ins:trumentan los compromisos por pensiones de las empresas con los trabajadores.

\section{El Sistema de la Seguridad Social.}

Para el cumplimiento del mandato constitucional de su artículo 41 el sistema de la Seguridad Social garantiza a todas las personas comprendidas en su campo de aplicación y a sus familiares y asimilados a su cargo, la posibilidad de obtener una asistencia sanitaria y unas prestaciones económicas que sustituyan las rentas que han dejado de percibir como consecuencia de haberse producido alguna de las situaciones protegidas que dan derecho a esas prestaciones, como pueden ser la jubilación, incapacidad temporal y permanente, maternidad, desempleo, fallecimiento. Para poder satisfacer estas prestaciones, la Seguridad Social, cuenta con una serie de recursos, siendo, las cuotas satisfechas por los empresarios y los trabajadores las que nos interesa en este momento.

\section{LAS COTIZACIONES O APORTACIONES}

A efectos del Impuesto sobre la Renta de las Personas Físicas las cotizaciones a la Seguridad Social que son a cargo de los trabajadores que previamente ha sido descontadas por su empresario en el momento de hacerles efectivas sus retribuciones, tienen para el trabajador la calificación de gasto deducible de sus rendimientos del trabajo [artículo 17.3.a) LIRPF]. Mientras para el empresario será gásto deducible de su actividad (en el IRPF, sí se trata de un empresario persona física 0 en el Impuesto sobre Sociedades, sí el empresario es una persona jurídica) , tânto, la parte de cuota que corresponde a los trabajadores, como, la parte de la cotización que corresponde al empresario.

\section{LAS PRESTACIONES}

En relación con las prestaciones que pueden obtenerse a cargo de la Seguridad Social se califican de forma expresa como rendimientos del trabajo, asi, en estesen- 
tido, el artículo 16.2.a) 1. ${ }^{a}$ de la LIRPF preceptúa que en todo caso tendrán la consideración de rendimientos del trabajo, "las pensiones y baberes pasivos percibidos de los regímenes públicos de la Seguridad Social y clases pasivas y demás prestaciones priblicas por situaciones de incapacidad, jubilación, accidente, enfermedad, viudedad, orfandad o similares". En cuanto a este tipo de prestaciones, debemos de tener en cuenta, que en algunas de ellas, desde el momento en que comienza a percibirse, se encuentra exentas del impuesto, como son las del artículo 7 en sus letras c) *las pensiones reconocidas en favor de aquellas personas que sufrieron lesiones o mutilaciones con ocasión o como consecuencia de la Guerra Civil 1936/1939, f) "las prestaciones reconocidas al contribuyente por la Seguridad Social o por las entidades que la sustituyan como consecuencia de incapacidad permanente absoluta o gran invalidez", y h) "las prestaciones familiares por bijo a cargo".

Estos rendimientos del trabajo deben ser declarados por el perceptor de la pensión, con independencia de que la persona que ha generado el derecho a su percepción sea distinta de la del beneficiario de la misma, por tanto, deben ser declaradas por la persona física en cuyo favor estén reconocidas, (artículo 11.2 de la LIRPF).

Para este tipo de prestaciones, cuando comienza a recibirlas el contribuyente, la Ley ha establecido una serie de particularidades a la hora determinar cual es el importe integro de estas pensiones calificadas como rendimientos del trabajo, que podemos resumir de la siguiente forma:

a)- la prestación la percibe en forma de renta; en este caso, el importe íntegro del rendimiento del trabajo, será la totalidad de lo recibido durante el período impositivo. [artículos 16.2.a) $1^{\text {a }}$, y 17.2.e)]

b)- la prestación, en el caso de obtenerse, en forma de capital:

Las prestaciones por situaciones de jubilación, viudedad, orfandad o similares, seguirán siendo rendimientos del trabajo, [articulo 16.2.a).1 $1^{a}$ del la LIRPF], pero su importe íntegro, no será la totalidad de la renta recibida en un pago único sino el resultado de practicar una reducción. Para cuantificar el importe de la reducción se toma como base la totalidad de la prestación recibida en forma de capital y, sobre esa base, se practicará una reducción del 40 por 100 , pero para tener derecho a practicar dicha reducción, es necesario que hayan transcurrido más de dos años desde la primera aportación que da derecho a obtener la pensión de jubilación. Esto significa, que como consecuencia de la aplicación de la reducción, el importe íntegro de la pensión de jubilación, obtenida en forma capital, la cantidad a imputar, será solamente del 60 por 100, [artículo 17.2.b) de la LIRPF]. 
En el caso de prestaciones por invalidez, nos podemos encontrar con presta $\rightarrow$ : ciones por incapacidad permanente absoluta o gran invalidez, que están exentasir en su totalidad, [artículo 7.f) de la LIRPF]. Para aquellas prestaciones, que no le es: de aplicación dicho supuesto de exención, como pueden ser la incapacidad pát: cial o total, al estar gravados como rendimientos del trabajo, su importe integro sera el obtenido tras practicarle sobre la totalidad de la prestación una reducción 40 pót 100 , pero con la particularidad, que en este caso no es necesario que haya trans: currido dos años para tener derecho a la reducción, por lo que se imputará el 60. por 100 de la prestación [artículo 17.2.b) de la LIRPF].

Para los trabajadores acogidos al régimen especial de trabajadores autónømøs (RETA) de la Seguridad Social, en cuanto a las cuotas satisfechas serán, gasto deducible de la actividad económica, mientras que las prestaciones que puedan recibir: de la Seguridad Social tienen el mismo tratamiento tributario que hemos comentando anteriormente.

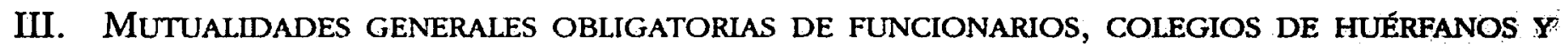
OTRAS INSTITUCIONES SIMILARES

Para garantizar la adecuada protección o cobertura frente los riesgos y contingencias que les puede sobrevenir a los funcionarios vinculados con la Administración Pública correspondiente, se crearon las Mutualidades generales de los funcionarios, como pueden ser, la MUFACE, MUMPAL, ISFAS, MUGEJU, que son sistemas de previsión social sustitutorios y no complementarios de la Seguridad Social, financiándose en parte por las cotizaciones de los funcionarios o mutualistas

Como su finalidad es coinciden con la de los distintos regímenes de la Seguridad Social, el impuesto que comentamos otorga un mismo tratamiento a ambos, tanto para cotizaciones de los mutualistas como, para las prestaciones que garantizan estas Mutualidades.

\section{LAS CUOTAS O APORTACIONES}

En relación con las aportaciones o cotizaciones efectuadas por los mutualistas, se computarán como gasto deducible de su rendimiento del trabajo, [artículo 17.3.a), b) c) de la LIRPF]. 


\section{LAS PRESTACIONES}

Cuando reciben algunas de las prestaciones, el tratamiento tributario es el mismo que en el caso anterior, es decir, se tratará de rendimientos del trabajo, lartículo 16.2.a).2. ${ }^{a}$, de la LIRPF], aunque tienen derecho a aplicar una reducción del 40 por 100 sobre la totalidad de la prestación, siempre que se reciba en forma de capital, [artículo 17.2.b) de la LIRPF], y siempre que haya transcurrido más de dos años desde la primera aportación, salvo, para las prestaciones por invalidez, que no es necesario el transcurso de este tiempo para tener derecho a la reducción.

Para estas mutualidades la norma no indica cuales son las contingencias que deben de acaecer y que están garantizadas a través de prestaciones, a diferencia de lo que ocurre con el sistema de la Seguridad Social. Como consecuencia de esta omisión, solamente tendrán el tratamiento fiscal descrito anteriormente, aquellas contingencias que quedan cubiertas al integrarse de forma obligatoria en la mutualidad, siempre que sean similares a las protegidas por la Seguridad Social, quedando excluidas las demás.

Además, estarán exentas las pensiones por inutilidad o incapacidad permanente del régimen de clases pasivas, siempre que la lesión o enfermedad que hubiera sido causa de las mismas, inhabilitare por completo al perceptor de la pensión para toda profesión u oficio [artículo 7.g) de la LIRPF].

En resumen: toda la renta obtenida por el contribuyente a lo largo del período impositivo que se destina a la financiación de los sistemas públicos de pensiones tiene la consideración de gasto deducible de sus rendimientos del trabajo, lo cual significa que no se tributa por esta renta en el momento de su percepción. Como consecuencia de lo anterior, será cuando comience a recibir la prestación cuando tributará como rendimientos del trabajo personal.

IV. Planes de Pensiones. [ART.17.2.A).3. ${ }^{a}$ DE LA LiRPF]

\section{APUNTES SOBRE SU RÉGIMEN JURIDICO}

La normativa que regula esta institución es la Ley $8 / 1987$, de 8 de junio, de Planes y fondos de Pensiones, desarrollada por el Real Decreto 1307/1988, de 20 de septiembre, que aprueba su Reglamento, aunque ambas, han experimentado numerosas modificaciones. Antes de analizar el régimen tributario de las aportaciones efectuadas y las prestaciones concedidas a los beneficiarios de un Plan de Pensiones, es necesario, efectuar una serie de consideraciones previas: 
- Los Planes de Pensiones se configuran como instituciones de previsión voluni taria y libre, cuyas prestaciones, de carácter privado, no serán, en ningún caso, sự de titutivas de las preceptivas del sistema público, pudiendo ser complementarias o no de aquellas. A través de estos Planes se define el derecho de las personas a perét bir rentas o capitales por jubilación o situación asimilable [artículo 16:1 Reglamento de Planes y Fondos de Pensiones, en la redacción dada por el Real Decreto 1589/1999, de 15 de octubre], viudedad, orfandad o invalidez, donde se distinguen los siguientes elementos personales: los sujetos constituyentes: el pros: motor del plan, teniendo tal consideración cualquier entidad, corporación, socie: dad, empresa, asociación, sindicato o colectivo de cualquier clase que insten a sù creación o participen en su desenvolvimiento; los participes, aquellas personas físis cas a cuyo interés se crea el plan, con independencia de que realicen o no apoitaciones. Por último, los beneficiarios, que son las personas físicas con derecho a la percepción de las prestaciones, hayan sido o no partícipes.

- Según el artículo 4 de la Ley 8/1987, se pueden distinguir tres mødalidades de planes de pensiones atendiendo a los sujetos constituyentes: a) sistema empleo, en este caso, el promotor es cualquier entidad, corporación, sociedad o empresa cuyos partícipes son los empleados. El promotor sólo podrá serlo de un plan de pensiones, al que exclusivamente podrán adherirse como partícipes los empleados. de la empresa promotora, aunque cabe la posibilidad de planes conjuntos por varias empresas, [Capítulo X del Reglamento de Planes y Fondos de Pensiones, incorporado por el Real Decreto 1589/1999, de 15 de octubre]. Las aportaciones pueden ser efectuadas tanto, por el promotor, que será lo normal, como, por los partícipes, es decir, los empleados. b) Sistema asociado, corresponde a planes curyo promotor es cualquier asociación, sindicato, gremio, colectivo, siendo los partícipes sus asociados y miembros, con la particularidad de que solamente pueden efectuar aportaciones las partícipes -es el sistema menos utilizado-. C) Sistema individual, el promotor será una o varias entidades de carácter financiero y cuyos partícipes son cualquier persona física, pudiendo efectuar aportaciones solamentè los partícipes que se adhieren al plan de pensiones -es la modalidad más extendida en la actualidad-.

Por otra parte, atendiendo a las obligaciones estipuladas, los planes de pensiones se podrán ajustar las modalidades siguientes: a) planes de aportación definidia. en estos se acuerda en cada momento la cuantía de las contribuciones de los promotores y, en su caso, de los partícipes al plan. Con esta modalidad se produce una incertidumbre sobre el importe de la prestación a recibir, que se cuantifícará en el momento de producirse la contingencia, que será iguál a la suma de tọ las las aportaciones efectuadas más los rendimientos que se hayan generađo. bj) Planes de prestación definida, en este caso, lo que se predetermina no es el importe de 
lass aportaciones sino el importe de la prestación a percibir por los beneficiarios, siendo necesario calcular, con cierta regularidad, las contribuciones necesarias para poder obtener cuando se produzca la contingencia el importe de la prestación predefinido. c) Planes mixtos, cuyo objeto es, simultáneamente, la cuantía de la prestación y la cuantía de la contribución. Los planes de los sistemas de empleo y asociados podrán ser de cualquiera de las tres modalidades y los del sistema individual, sólo de la modalidad de aportación definida.

- Para comprender mejor el tratamiento fiscal de los planes de pensiones es necesario recordar que la finalidad básica de los mismos consiste en facilitar el bienestar futuro de la población retirada mediante la obtención de prestaciones complementarias a las de la Seguridad Social que permitan mantener un nivel de vida parecido al que se tenía hasta el momento de producirse la contingencia protegida que ha conllevado dejar de obtener una serie de rentas. Además, es un importante elemento que ayuda al fortalecimiento del sistema financiero, debido a que su implantación pone de manifiesto una modalidad de ahorro a largo plazo, prácticamente, durante toda la vida activa de una persona, mediante las aportaciones efectuadas al plan, no pudiendo hacer uso de las aportaciones como de su rentabilidad, hasta que se produzcan las contingencias que dan derecho a las prestaciones, salvo en los supuestos de enfermedad grave o desempleo de larga duración, que permiten hacer efectivos los derechos consolidados.

Pues bien, para favorecer la implantación de los planes de pensiones se les otorga un tratamiento favorable consistente en el diferimiento de la tributación de las contribuciones, es decir, tanto las aportaciones como la rentabilidad que generan en el fondo de pensiones "quedan excluidos de gravamen", tributando en un momento posterior, cuando se perciben las prestaciones del Plan de Pensiones, que sí están gravadas.

\section{Regimen tributario de las aportaciones del promotor al Plan de Pensiones}

En el plan sistema empleo las aportaciones pueden ser efectuadas tanto por el promotor, como por el partícipe. En relación con las aportaciones del promotor (empleador) en favor de los partícipes (empleados), el artículo 27 de la Ley 8/1987 dispone que la contribuciones de los promotores serán deducibles en el impuesto personal que grava su renta, es decir, será gasto deducible en el Impuesto sobre Sociedades, en el caso de personas jurídicas, o en el IRPF, si se trata de personas físicas que ejercen de actividades económicas. Siendo imprescindible que se impute a cada partícipe del Plan de Pensiones la parte que le corresponda sobre las citadas contribuciones. 
Para el participe, la parte que le ha sido imputada de las aportaciones del promo tor deberá integrarla en su base imponible del IRPF, que de acuerdo con el artículó 16.1.e) de la LIRPF se califican como rendimientos del trabajo, y el artículo 44.1.1. $\mathrm{G}$ ) de la misma Ley los cataloga como rentas en especie, que se valorará por el importer imputado al participe, quedando exonerado el promotor de tener que practicar el ingreso a cuenta, basándose en el artículo 95.2 del RIRPF. Pero como analizaremos â continuación estos rendimientos del trabajo en especie obtenidos e imputados al partticipe están "exoneradas de tributación" debido a que tienen la posibilidad de redueiri su base imponible de acuerdo con unos requisitos y límites que analizaremos a con: tinuación.

\section{RÉGIMEN TRIBUTARIO DE LAS APORTACIONES DEL PARTICIPE}

\subsection{REGGIMEN GENERAL}

Las aportaciones de los promotores imputadas a los participes, calificadas como rendimientos del trabajo en especie, junto con las aportaciones directas que pueden efectuar los propios participes al plan de pensiones, en la modalidades sistema empleo, quedarán "exoneradas de tributación" parcial o totalmente, al poder reducir su base imponible del IRPF por el importe de las aportaciones, de acuerdo con deducción prevista en el artículo 46 de la Ley de dicho impuesto. Algo parecido ocurre con las contribuciones efectuadas por los participes a los Planes de Pensiones en sus modalidades del sistema asociado, como del sistema individual, ya que en estas modalidades no cabe la posibilidad de aportaciones por parte del promotor, pudiendo diferir la tributación de las rentas destinadas al plan a traveés de la reducción de la base imponible del impuesto personal del participe.

Para poder practicar esta reducción de la base imponible, a parte de requisitos de carácter formal, no podrá superar un límite cuantitativo, es decir, se establece como límite máximo de la reducción, la menor de las siguientes cantidades:

a) el 20 por 100 de la suma de rendimientos netos del trabajo y de actividades económicas percibidos individualmente durante el ejercicio;

b) 1.100 .000 pesetas anuales.

En los casos de tributación conjunta, el límite máximo se aplica individualmen:te para cada uno de los participes de la unidad familiar [artículo 70.2.10 de la LIRPF] y se debe de computar de manera conjunta, para la determinación del línîte de la reducción, las aportaciones que se hayan podido efectuar a mutualidades de previsión social y planes de pensiones. 


\subsection{REgÍMENES PARTICULARES}

\subsubsection{Personas con minusvalía igual o superior al 65 por 100}

En la Disposición Adicional Decimoséptima de la LIRPF y el art.10.ter del Reglamento de Planes y Fondos de Pensiones incorporado por el Real Decreto 215/1999, se establece el régimen jurídico específico para las aportaciones y prestaciones de planes y fondos de pensiones y mutualidades de previsión social relativas a personas con un grado de minusvalía igual o superior al 65 por 100, que a partir del 1 de enero de 1999 las aportaciones realizadas podrán ser objeto de reducción en la parte general de la base imponible del IRPF, con los siguientes límites:

- las aportaciones anuales realizadas por cada partícipe a favor de personas con minusvalía (designado beneficiario) con las que exista relación de parentesco en línea directa o colateral hasta el tercer grado inclusive, con el límite de 1.100 .000 pesetas anualès. Estas aportaciones no estarán sujetas al Impuesto sobre Sucesiones y Donaciones. Ello sin perjuicio, a parte, de las aportaciones que puedan realizar a sus propios planes de pensiones por cada partícipe.

- las aportaciones anuales realizadas por las personas minusválidas participes, con el límite de 2.200 .000 pesetas anuales.

Pero para los anteriores límites hay que tener en cuenta las siguientes reglas para su determinación:

- las aportaciones anuales máximas realizadas a favor de una persona con minusvalía, incluyendo las propias aportaciones de la persona con minusvalía no podrá rebasar la cantidad de 2.200 .000 pesetas.

- el conjunto de las reducciones practicadas por todas las personas que realicen aportaciones a favor de un mismo minusválido, incluidas las del propio minusválido, no podrá exceder de 2.200 .000 pesetas anuales. Por tanto, si el minusválido ha efectuado el máximo de la aportación, los restantes participes que han efectuado aportaciones no podrán practicarse reducción alguna en la parte general de se base imponible. Si no ha alcanzado el tope máximo, si podrá efectuar dicha reducción teniendo en cuenta el límite individual y el conjunto de 2.200 .000 pesetas.

- en el caso de ser varias las personas que efectúan aportaciones en favor del minusválido, habrán de ser objeto de reducción, en primer lugar, las aportaciones realizadas por el propio minusválido y sólo si las mismas no alcanzaran el límite de 2.200.000 pesetas señalado, podrán ser objeto de reducción las aportaciones realizadas por otras personas a su favor en la base imponible de éstas, de forma 
proporcional, sin que en ningún caso el conjunto de las reducciones practicadas por todas las personas que realizan aportaciones a favor de un mismo minusvallido pueda exceder de 2.200 .000 pesetas, incluyendo también las propias aportaciones efectuadas por el minusválido.

- en este caso no es de aplicación el límite porcentual especificado en el régít: men general.

- las personas con una relación de parentesco con el minusválido podrán redứcir la parte general de su base imponible por dos conceptos compatibles y con limites distintos, primero, el régimen general en relación con las aportaciones efectuadas a sus propios planes de pensiones y, segundo, en relación con las aportaciones efectuadas a favor del plan de pensiones de la persona minusválida.

- los límites recogidos en la Disposición adicional decimoséptima serán conjuntos para las aportaciones de Planes de Pensiones y a Mutualidades de Previsión Social.

\subsubsection{Personas mayores de 52 años}

El artículo 5.3 de la Ley de Planes y Fondos de Pensiones según la nueva redacción dada por la Ley 66/1997, de 30 de diciembre de Medidas Fiscales, Administrativas y del Orden social establece que las aportaciones máximas a los planes de pensiones no podrá rebasar en ningún caso la cantidad de 1.100:000 pesetas, aunque autoriza para que reglamentariamente se establezcan cuantias superiores para aquellos partícipes, a los que por su edad, dicha cantidad les resulte insuficiente. En base a dicha autorización, se da nueva redacción al articulo 13 del Reglamento de Planes y Fondos de Pensiones, por el Real Decreto $1589 / 1999$, de 15 de octubre, por el que las aportaciones máximas anuales que pueden efectuar las personas que superen la edad de 52 años podrá ir inerementado su aportación por cada año de más que tenga el trabajador, como a continuación se indica: 


\begin{tabular}{|c|c|c|c|}
\hline Edad & Límite inicial & Limite adicional & Límite final \\
\hline 52 & 1.100 .000 & 0 & 1.100 .000 \\
\hline 53 & 1.100 .000 & 84.615 & 1.184 .615 \\
\hline 54 & 1.100 .000 & 169.231 & 1.269 .231 \\
\hline 55 & 1.100 .000 & 253.846 & 1.353 .846 \\
\hline 56 & 1.100 .000 & 338.462 & 1.438 .462 \\
\hline 57 & 1.100 .000 & 423.077 & 1.523 .077 \\
\hline 58 & 1.100 .000 & 507.692 & 1.607 .692 \\
\hline 59 & 1.100 .000 & 592.308 & 1.692 .308 \\
\hline 60 & 1.100 .000 & 676.923 & 1.776 .923 \\
\hline 61 & 1.100 .000 & 761.538 & 1.861 .538 \\
\hline 62 & 1.100 .000 & 846.154 & 1.946 .154 \\
\hline 63 & 1.100 .000 & 930.769 & 2.030 .769 \\
\hline 64 & 1.100 .000 & 1.015 .385 & 2.115 .385 \\
\hline 65 en adelante & 1.100 .000 & 1.100 .000 & 2.200 .000 \\
\hline
\end{tabular}

De esta forma los participes mayores de 52 años podrán incrementar sus aportaciones a los planes de pensiones, no distinguiendo la normativa entre los de sistema empleo, individual o asociado, pudiendo duplicarse su aportación cuando se cumplan los 65 años hasta los 2.200 .000 pesetas. Este incremento de aportaciones se podrán iniciar durante el ejercicio de 1999 pudiendo reducir la parte regular de la base imponible del impuesto personal del participe pero teniendo en cuenta el otro límite fijado en la norma fiscal del 20 por 100 de la suma de los rendimientos netos del trabajo y de actividades económicas percibidos individualmente en el ejercicio, es decir, haber obtenido durante el ejercicio fiscal unos rendimientos netos mínimos de 11.000 .000 de pesetas.

Estas nuevas reducciones son posibles en la base imponible del Impuestos sobre la Renta de las Personas Físicas del partícipe como consecuencia de la remi- 
sión que efectúa la Ley de ese impuesto a la normativa de los Planes y Fondos dé Pensiones, con el siguiente tenor en el artículo 46.1.4' último párrafo: «El límite máximo de reducción fiscal dependerá del límite financiero que en cada caso se fije, en virtud de lo establecido en el artículo 5.3 de la Ley 8/1987, de 8 de junio, dé Regulación de los Planes y Fondos de Pensiones".

3.2.3. Régimen transitorio de las aportaciones efectuadas por el participe de planes de pensiones con posterioridad al cese de su actividad laboral y hasta la entrada en vigor de la Ley

En la Disposición Transitoria decimotercera de la LIRPF se recoge el tratamiento fiscal que debe de otorgarse a los derechos consolidados correspondientes a las aportaciones efectuadas con posterioridad al acaecimiento de la contingencia de jubilación del participe y antes de la entrada en vigor de la nueva Ley del Impuesto sobre la Renta de las Personas Físicas, es decir, antes del 1 de enero de 1999, con independencia de que el plan de pensiones se haya suscrito con posterioridad al cese de la vida laboral. Esta situación anómala se producía como consecuencia de que los beneficiarios de los planes de pensiones no realizaban la reclamación de la prestación por jubilación en el momento en que ésta acaecía, sino que dejaban: que el fondo acumulado seguirá produciendo más rentabilidad e incluso efectuando nuevas aportaciones con posterioridad a dicho momento.

Ante esta situación habrá que distinguir dos situaciones: primero, la descrita en dicha DT $13^{\circ}$, es decir, en relación con los derechos consolidados que corresponda a las cantidades aportadas con posterioridad a la jubilación y hasta el 31 de diciembre de 1998; segunda, los derechos consolidados derivados de las aportaciones efectuadas antes de la jubilación y que todavía no se hayan recuperado.

En relación con la primera se concede al participe la posibilidad de optar entre:

a) recuperar los derechos consolidados correspondientes a dichas aportaciones en forma de capital, tributando como rendimientos del trabajo y, gozando de una reducción del 40 por 100 recogida en el artículo 17.2.b) de la LIRPF, siempre que hubieran transcurrido más de dos años desde la primera aportación al plan de pen siones hasta el 31 de diciembre de 1998.

b) destinar las cantidades aportadas al plan una vez producida la jubilación, -por tanto, sus derechos consolidados- a cubrir aquella contingencia que aún no se ha producido y que se puede producir en el futuro, como es el fallecimiento del participe. Situación interpretada por la Dirección General de Tributos en su Consulta de 22 de marzo de 1999, en el siguiente sentido: "De acuerdo con el criterio sustentado por la Dirección General de Seguros, dada la finalidad de los planes de pensiones, configurados pro la Ley como instituciones de previsión destina. 
das a la consecución de prestaciones al acaecimiento de determinadas contingencias, se entiende que no procede realizar aportaciones para la cobertura de contingencias que ya se ban producido o que no pueden producirse o reiterarse.

Con carácter general, un jubilado no puede ser declarado inválido para la actividad laboral (no está expuesto al riesgo de invalidez para la actividad laboral en los distintos grados reconocidos por la Seguridad Social), ni tampoco puede volver a jubilarse (no tendrá un nuevo acceso a tal situación de jubilación salvo que reanude la actividad laboral o profesional causando alta en la Seguridad social de cara a una segunda jubilación). La única contingencia susceptible de acaecimiento será el fallecimiento.

En consecuencia, las personas jubiladas no podrân realizar aportaciones en concepto de jubilación o invalidez, aunque si podrán efectuar aportaciones para cubrir la contingencia de fallecimiento....

Esta opción se debe ejercitar antes del 31 de diciembre de 1999, y ante la falta de manifestación expresa, se entiende haber optado por el mantenimiento de los derechos coǹsolidados para cubrir la contingencia de fallecimiento. Además, no cabe la posibilidad de recuperar parte de los derechos consolidados y dejar el resto para cubrir la contingencia de fallecimiento, ya que el ejercicio de la opción debe ser por la totalidad de los derechos consolidados derivados de las aportaciones efectuadas con posterioridad del cese de la actividad laboral

En relación con la segunda situación, que no le es de aplicación lo recogido en la DT $13^{\circ}$, es decir, los derechos consolidados de las aportaciones efectuadas antes de la jubilación que todavía no se han recuperado, que deberían haberse recuperado a partir del momento en que se hubiera producido la contingencia. Ante el silencio de la LIRPF sobre esta situación, la Dirección General de Tributos en consulta de 22 de marzo de 1999 considera que: "De acuerdo con el criterio sustentado pro la Dirección General de Seguros, el participe en un breve plazo con posterioridad al acaecimiento de la contingencia (en este caso de la jubilación) debe comunicar y acreditar su acaecimiento a la Entidad Gestora y definir la forma en que desea percibir la prestación, de entre las previstas en las especificaciones del Plan y siempre conforme a lo establecido en la normativa. Además, al señalar la forma de percibir la prestación, puede (si asi está previsto en las especificaciones del Plan) elegir percibir la prestación de manera inmediata o diferida puesto que la normativa vigente no exige la percepción del capital, renta o una combinación de ambas sea inmediata a la fecha de la contingencia, pro lo que podría ser diferida a una fecha determinada posterior... Por tanto, el beneficiario deberá determinar la forma en que desea percibir la prestación en el momento de causarse la contingencia, dentro de las previstas en la Ley y en las propias especificaciones del Plan. Cualquier otra fórmula distinta que conlleve prestaciones a recibir con total flexi- 
bilidad por parte del beneficiario presupone una total disponibilidad de la presta ción y debe entenderse como reconocimiento del derecho económico a favor del beneficiario, en el momento del acaecimiento de la contingencia; en consecuencia;. se produciria el devengo de la totalidad de la prestación en dicbo momento, con: independencia del momento del cobro".

De acuerdo con esta interpretación surge el problema de qué ocurre en aquellos. casos en que el ejercicio en que se produjo la contingencia de jubilación ha preseñto y no se comunicó y acreditó su acaecimiento a la Entidad Gestora. Ante esta situación caben básicamente dos posturas, aquella que considera que si hubieran transcurrido más de cuatro años desde la fecha de la jubilación y se lleva a cabo la recuperación del capital, no tributaría, por haber prescrito los derechos consolidados debido que la interpretación administrativa sitúa el devengo en el momento de la contingencia de jubilación, con independencia del momento del cobro, ya que a partir de ese momento debe entenderse reconocido el derecho y por tanto, se presupone una total disponibilidad de la prestación por parte del beneficiario. En caso contrario, es decir, no han trascurrido más de cuatro años desde el momento de la. jubilación y se recuperan los derechos consolidados se aplicará el régimen general de las prestaciones por planes de pensiones, aplicándose las sanciones e intereses de demora correspondientes, por haber dejado de ingresar en el ejercicio en que se produjo el devengo de dicha prestación. Aunque en este caso, consideramos que puede optar por mantener los derechos consolidados de las aportaciones efectuadas antes de producirse la jubilación para cubrir la contingencia de fallecimiento.

Una segunda tesis, que consideramos la correcta, según la cual, el partícipe una vez que se produce la contingencia nace el derecho a percibir la correspondiente prestación, teniendo la opción de poder eligir cuando serán exigibles, es decir; que el pago de la prestación sea inmediato a la fecha de la contingencia o diferido:a un momento posterior, pero si éste no comunica dicha situación a la Entidad Gestora y trascurren más de cuatro años en relación con el momento que decide recuperar los derechos adquiridos no consideramos que se haya producido la prescripción ya que el beneficio concedido al participe para que decida cuando podrá ser exigible dicha prestación no podrá obtener el beneficio de la prescripción coño consecuencia de incumplir el deber de comunicar dicha situación a la Entidad. Gestora. En este caso consideramos que la prestación nace a partir del momento en que el partícipe decide cuando se hará exigible, por tanto, será a partir de ese momento cuando habrá que iniciar el cómputo de la prescripción y no a partí̀ del momento en que ha acaecido la contingencia.

Esta situación no se va a repetir en el futuro ya que tras la modificacion del Reglamento de Planes y Fondos de Pensiones por el Real Decreto 1598/1999/re 15 de octubre, se establece un plazo máximo de seis meses para que los partiópés 
comuniquen a la entidad gestora el acaecimiento de la contingencia que da derecho a la prestación indicando el cómo se efectuará el cobro de la prestación y el cuando se va ha efectuar el rescate del plan, es decir, las sumas de las aportaciones y los rendimientos generados, a partir de que momento se desea comenzar a recibirlos y en el caso, de que llegado el vencimiento, el beneficiario se opone al cobro del capital, o no señalase el medio de pago, la entidad gestora depositará su importe en una entidad de crédito a disposición y por cuenta del beneficiario, entendiéndose así satisfecha la prestación a cargo del Plan de Pensiones, todo ello, de acuerdo con el nuevo articulo 16.bis incorporado al Reglamento de Planes y Fondos de Pensiones efectuada por el Real Decreto 1589/1999, de 15 de octubre.

Como consecuencia de las reducciones comentadas la parte de renta obtenida a lo largo del ejercicio que se ha ahorrado destinándola al Plan de Pensiones "no tributan en ese ejercicio, sino que tributará en el momento que se comience a percibir la correspondiente prestación del Plan. momento, que como consecuencia de la contingencia, el contribuyente suele tener unos menores ingresos que los obtenidos a los lárgo de su vida laboral, por lo que el gravamen efectivo que recaerá sobre las prestaciones podrá ser inferior que el que soportaría en el momento en que se obtuvieron, como consecuencia de la progresividad del impuesto.

Por tanto, en aquellos casos en que el importe de las aportaciones efectuadas al plan de pensiones sea una cantidad superior a la del límite anterior, el exceso entre la aportación y el límite máximo de reducción, sí se encontrará gravado. Pero para evitar esta situación cabe dos posibilidades:

Primera. Recogida en la nueva redacción del art.13 del Reglamento de Planes y Fondos de Pensiones otorgada por el Real Decreto 1589/1999, de 15 de octubre, según la cual si las aportaciones efectuadas por el participe (él mismo o su empresa) a uno o varios planes de pensiones superan los límites máximos establecidos en la normativa reguladora de los Planes de Pensiones, es decir, 1.100 .000 pesetas con carácter general, o las efectuadas por participes minusválidos (art.10.ter del Reglamento de Planes de Pensiones), o por mayores de 52 años (art. 13.2, nueva redacción del Regl. PFP, dada por el RD 1589/1999) se podrá retirar el exceso que se produzca sobre las aportaciones máximas antes del 30 de junio del año siguiente, de acuerdo con el procedimiento descrito en el art. 13.4 del Regl. PFP, en la redacción dada por el RD 1589/1999.

Segunda. Si el exceso proviene no del hecho de haber superado los límites máximos recogidos en la normativa de los Planes y Fondos de Pensiones, sino por haber superado el límite máximo de reducción recogido en la normativa del IRPF, de acuerdo con la nueva redacción del artículo 27 de la Ley 8/1987, como en el artículo 50 del RIRPF cabe la posibilidad, previa solicitud, que el exceso que no ha podido reducir la base imponible pueda serlo en los cinco ejercicios siguientes: 
"La solicitud deberá realizarse en la declaración del Impuesto sobre la Renta de las. Personas Físicas correspondiente al ejercicio en que las aportaciones realizadas bayan. excedido de los limites antes mencionados.

El exceso que, de acuerdo con lo señalado, no haya podido ser objeto de reducción, se imputará al primer ejercicio, dentro de los cinco ejercicios siguientes, en que las aportaciones efectuadas no alcancen los limites cuantitativos establecidos. en el artículo 46.1 de la Ley del Impuesto.

Cuando concurran aportaciones realizadas en el ejercicio con aportaciones de ejercicios anteriores que no bayan podido ser objeto de reducción por exceder de los: limites establecidos, se entenderán reducidas, en primer lugar, las aportaciones correspondientes a años anteriores.

De esta forma se trata de evitar la doble imposición que se puede producir como consecuencia de gravar la parte de renta destinada como aportación al plan y que ha superado el límite máximo de reducción de la base imponible, para posteriormente, gravar como rendimiento del trabajo las prestaciones, cuyo importe va venir definido por las cantidades de las aportaciones efectuadas (que parte de ellas, al no beneficiarse de la reducción, tributaron en su momento), más la rentabilidad que hayan generado durante su permanencia en el Fondo de Pensiones.

¿Pueden efectuar aportaciones al Plan de Pensiones los participes que no obtengan rendimientos del trabajo y/o rendimientos de actividades económicas?. La respuesta debe ser afirmativa, es decir, el participe puede efectuar aportaciones que pueden tener su origen en otro rendimiento, como puede ser del capital mobiliario, inmobiliario o ganancias patrimoniales, pero en estos casos, estas aportaciones quedan excluidas de la reducción, al superar siempre el límite máximo percentuàl de reducción fijado en el artículo 46 de la LIRPF. Aunque de esta forma se establece una discriminación que en algunos casos no consideramos razonable, como ocurre en los matrimonios que solamente uno de los dos cónyuges obtiene rentas del trabajo o de actividades económicas, ya que no podrá practicar la correspondiente reducción el otro cónyuge, por lo que se debería haber dado la posibilidad para poder aplicarse la misma, ya que cuando se produzca la contingencia, no exis:ten garantías de que no se vean alteradas las fuentes de renta necesarias para atender a la subsistencia, como ocurre en las personas que únicamente obtienen elevados rendimientos del capital..

4. RÉGIMEN TRIBUTARIO DE LAS PRESTACIONES PERCIBIDAS POR LOS BENEFICIARIOS DE LOS: Planes de Pensiones

Los beneficiarios de los planes de pensiones, una vez acaecida la contingenda 
podrán elegir la forma en que van a percibir sus prestaciones, entendiendo por prestación el derecho económico en favor de los beneficiarios de un plan de pensiones, como resultado del acaecimiento de una contingencia cubierta por el citado plan, (art.16.1 del Regl. PFP, en su nueva redacción dada por el RD 1589/1999), estableciéndose en el art. 16.bis. de dicho Reglamento, -incorporado por el RD 1589/1999- que la prestación podrá consistir en:

a) una prestación en forma de capital, consistente en una percepción de pago único. El pago de esta prestación podrá ser inmediato a la fecha de la contingencia o diferido a un momento posterior.

b) una prestación en forma de renta, consistente en la percepción de dos o más pagos sucesivos con periodicidad regular, incluyendo al menos un pago en cada anualidad. Las rentas podrán ser inmediatas a la fecha de la contingencia o diferidas a un momento posterior.

c) prestaciones mixtas, que combinan rentas de cualquier tipo como un único cobro en forma de capital.

Es deber del beneficiario o de su representante legal comunicar a la comisión de control del plan o a la Entidad Gestora del Plan el acaecimiento de la contingencia, señalando en su caso la forma elegida para el cobro de la prestación, junto la documentación que proceda según lo previsto en las especificaciones del plan, todo ello en un plazo no superior a seis meses desde que se hubiera producido la contingencia desde su reconocimiento por la autoridad u organismo correspondiente.

De acuerdo con lo anterior, será el beneficiario el que decida sobre el sistema de cobro de sus prestaciones, indicando el cómo y el cuando desean recuperar el ahoro que le ha generado dicho plan de pensiones. Esto no significa que a partir de ese plazo de seis meses va a comenzar a recibir la prestación, sino que durante ese periodo de tiempo debe de decidir cuando se va a iniciar su rescate, por tanto, su periodicidad, que puede ser al cabo de uno, dos, tres años....).

Esta posibilidad aparece descrita en el artículo 16.bis. del Regl. PFP hay que ponerla en relación con la regla de imputación temporal recogida en el artículo 14 de la LIRPF, según la cuál los rendimientos del trabajo -como son las prestaciones percibidas por los beneficiarios de los Planes de Pensiones [art. 16.2.a).3. ${ }^{a}$ LIRPF] se imputarán al período impositivo en que sean exigibles por su perceptor. En este caso, la normativa fiscal ha optado por el criterio de la exigibilidad, entendida como aquél momento en que el contribuyente puede exigir o demandar el pago de la deuda a su deudor. En el caso de las prestaciones derivadas de un Plan de Pensiones, para el beneficiario nace el reconocimiento del derecho a su percepción desde el mismo momento que se produce la contingencia, y este se produce, como regla general, a los 15 dias, como máximo, desde el momento en que el beneficia- 
rio comunica el acaecimiento de la contingencia, de acuerdo con la especificacio nes anteriores, o como recoge el art. 16.bis del Regl. PFP. Por tanto, el beneficiario deberá imputar la prestación no en el ejercicio en que nace el derecho a su petcepción sino en el ejercicio que él hubiera determinado, por tanto, diferirlo en el tiempo, de acuerdo con la modalidad de cobro que de la misma hubiera fijado en el contrato. A partir de ese momento es cuando nace la exigibilidad de la prestación por lo que en aquellos casos en que difiere el cobro a un momento posterior, la rentabilidad diferida, a parte de la particularidad de la Disposición Transitorio $13^{2}$, se calificará como rendimientos del capital mobiliario

Esta clase de prestaciones se podrán percibir cuando acontezca alguna de las siguientes contingencias protegidas por el plan de pensiones:

a) jubilación o situaciones asimilable;

b) invalidez laboral total y permanente para la profesión habitual o absoluta y permanente pata todo trabajo y la gran invalidez $y$ :

c) muerte del partícipe que puede generar el derecho a prestaciones de viudedad, orfandad, o en favor de otros herederos o personas designadas; en el caso de muerte del beneficiario que no hay sido previamente partícipe, únicamente se pueden generar prestaciones de viudedad $u$ orfandad.

Según lo anterior, el beneficiario que percibe las anteriores prestaciones coincide con el participe en el caso de jubilación o invalidez, pero no, en el caso de fallecimiento del partícipe, siendo el beneficiario una tercera persona, pero a pesar de esto, en todos los casos tendrán para el beneficiario de la prestación, exista o no. coincidencia con el participe, la calificación de rendimientos del trabajo, cualquiera que sea la condición del participe: trabajador, profesional o empresario, como dispone el artículo 16.2.a).3. ${ }^{2}$ de la LIRPF, por tanto, no se tributará por el Impuesto sobre Sucesiones o Donaciones.

A la hora de determinar el rendimiento íntegro de estos rendimientos del trabajo es necesario tener en cuenta las siguientes particularidades:

- si la prestación se recibe en forma de renta, su importe íntegro viene determinado por el importe de la misma obtenido a lo largo del período impositivo, [artículo 162.e) de la LIRPF].

En el caso de prestaciones obtenidas por personas con minusvalía en virtud de las aportaciones realizadas de acuerdo con la Disposición Adicional Decimoséptima.7, se calificarán como rendimientos del trabajo, gozando de una reducción en su IRPF hasta un importe máximo de dos veces el salario mínimo interprofesional.

- si la prestación se percibe en forma de capital, es decir, una percepción de pago único, el beneficiario tendrá derecho a aplicar una reducción del 40 por 100 
sobre el importe de la totalidad de la prestación percibida, siempre que hayan transcurrido más de dos años desde la primera aportación que da derecho a la prestación. Las prestaciones por invalidez gozan de dicha reducción sin necesidad de haber transcurrido dicho plazo de generación, aunque no podrá acogerse a la exención del artículo 7. f) y g) de la LIRPF.

Para las prestaciones recibidas en forma de capital por las personas con minusvalía, correspondientes a las aportaciones a las que se refiere la Disposición Adicional Decimoséptima.7 la reducción será del 50 pro 100, siempre que hayan transcurrido más de dos años desde la primera aportación.

- cuando la prestación se abona de manera mixta, que combine rentas de cualquier tipo con un único cobro en forma de capital, la reducción sólo resultará de aplicable al cobro efectuado en forma de capital, (artículo 11 del RIRPF).

Estas prestaciones, con independencia de la forma de percibirlas, no podrán minorarse por el importe de los excesos de las aportaciones o contribuciones que no pudieron minorarse en su momento en la base imponible del participe por haber superado los límites para ello.

V. Contratos de seguros concertados con mutualidades de Previsión Social. [ART.17.2.A).4. ${ }^{a}$ DE LA LIRPF].

Las mutualidades de previsión social son entidades aseguradoras que ejercen una modalidad complementaria, de carácter voluntario, al sistema de la Seguridad Social obligatoria, mediante aportaciones a prima fija o variable de los mutualistas, otorgando prestaciones en los supuestos de jubilación, viudedad, orfandad, invalidez, enfermedad o pérdida temporal del trabajo y desarrollando una labor social como pueden ser los servicios para la tercera edad, becas, ocio, etc. Entre las mutuas que agrupan a colectivos de profesionales colegiados podemos destacar a la Mutualidad General de la Abogacía, la de los Ingenieros Industriales o la de Aparejadores, o mutualidades de empleados, como la Mutualidad de empleados del Banco de España.

\section{APORTACIONES}

En cuanto al régimen fiscal de las aportaciones que se pueden efectuar a estas mutualidades podemos distinguir las siguientes situaciones:

a) Cuando se trata de profesionales no integrados en el régimen especial de la Seguridad Social de los trabajadores autónomos (RETA) o que ha optado por la 
Mutualidad, que ejercen su actividad con cuenta propia y el contrato de seguro coni la mutualidad actúe cono una alternativa al régimen especial anterior, tendrán lä consideración de gasto deducible de su actividad profesional la parte que tenga por objeto la cobertura de contingencias atendidas por la Seguridad Social, con el limin:-3 te máximo de 500.000 pesetas anuales, de acuerdo con el artículo 28 regla 1 : segundo párrafo de la LIRPF. En caso de exceso, éste, consideramos que se podra reducir en la base imponible del impuesto, como a continuación se expondrá.

b) Cuando se trata de mutualistas ejercientes por cuenta propia que ha optado: por RETA y por la Mutualidad, por tanto, actúa como un sistema complementario ali! anterior, tendrá la consideración de gasto deducible las cotizaciones efectuadas al RETA y, además, podrán reducir en su base imponible las cuotas abonadas a lä Mutualidad, con los límites a continuación se verán.

c) Cuando nos encontramos con contratos de seguro concertados con mutualidades de previsión social por trabajadores por cuenta ajena o socios trabajadores, incluidas las contribuciones del promotor que les hubiesen sido imputadas en con:cepto de en concepto de rendimientos del trabajo, de acuerdo con lo dispuesto en la disposición adicional primera de la Ley 8/1987 de 8 de junio, de regulación de os Planes y Fondos de Pensiones, que establece el régimen de protección de los compromisos por pensiones que las empresas asuman con los trabajadores, ya que se establece la posibilidad de que estos contratos, con las mutualidades, sean una alternativa en los procesos de exteriorización de los compromisos internos de las empresas por pensiones, desarrollado en el Real Decreto 1588/1999, de 15 de octubre, por el que se aprueba el Reglamento sobre la instrumentación de los compromisos por pensiones de las empresas con los trabajadores y beneficiarios.

Las aportaciones efectuadas a estas mutualidades darán derecho a la reducción de la base imponible del impuesto en los casos previstos en el artículo 46:1.1. ${ }^{\circ}$, . $^{\circ}$ y $3 .^{\circ}$ de la LIRPF y además, para poder llevar a cabo la reducción de la parte general de la base imponible es necesario que se den los siguientes requisitos, también recogidos en el artículo 46.1, letras a) a c) de la LIRPF: 1. Que las aportaciones máximas a tales contratos, tanto, las efectuadas por el mutualista, como, la imputadas por el promotor, no podrán superar el límite previsto para los Planes de Pensiones, 1.100.000 pesetas (art. 5.3 Ley 8/1987). 2. Los derechos consolidados de los mutualistas solamente se podrán hacer efectivos cuando concurran las mismas contingencias previstas para los Planes de Pensiones (art. 8.8 Ley 8/1987). 3. Las prestaciones percibidas tributarán, en su integridad, sin que en ningụ́n caso puedan minorarse en las cuantías correspondientes a los excesos de las contribuciónés sobre los límites de reducción en la base imponible. Límite de la reducción -con carácter general, a parte de las situaciones particulares-será lá menor de las dos 
cantidades siguientes: 20 por 100 de la suma de los rendimiento netos del trabajo y de actividades económicas percibidos individualmente en el ejercicio o 1.100 .000 pesetas anuales. Como puede observarse todas estas cautelas establecidas por el legislador para poder efectuar la reducción de la parte general de la base imponible viene motivada por hecho de que ha este tipo de contratos de seguro concertados con mutualidades de previsión social se les quiere dar el mismo tratamiento que a los Planes de Pensiones, y por este motivo, deben de tener las mismas o similares características que aquéllos.

En resumen: las cantidades abonadas en virtud de contratos de seguro concertados con mutualidades de previsión social, serán gastos deducible para los profesionales si se dan los requisitos del articulo 28.1." segundo párrafo de la LIRPF, mientras que para las demás situaciones descritas en el artículo 46.1 de la LIRPF, dichas cantidades podrán reducir la parte general de la base imponible, siempre que reúnan los requisitos recogidos en dicho artículo. Además, en aquellos casos, artículo $46.1 .3 .^{\circ}$ de la LIRPF, en que el contrato de seguro con la mutualidad se perfila como una àlternativa a la exteriorización de los compromisos de pensiones internos asumidos por las empresas, la contribución efectuada por el promotor y imputada al trabajador, tendrá para éste la consideración de rendimiento del trabajo en especie, [artículo 16.1.e) de la LIRPF], no sometida al sistema de ingresos a cuenta.

\section{Prestaciones}

En cuanto a las prestaciones obtenidas como consecuencia de estos contratos de seguro concertados con mutualidades de previsión social, el artículo 16.2.a) 4. ${ }^{\mathrm{a}}$ establece que en todo caso tendrán la consideración de rendimientos del trabajo personal, siempre que las cantidades abonadas hayan podido ser, al menos en parte, o, gasto deducible para la determinación del rendimiento neto de actividades económicas, u objeto de reducción en la base imponible del impuesto.

La integración de estas prestaciones, jubilación, invalidez o fallecimiento, como rendimientos del trabajo personal, se efectuará por su integridad, no pudiendo minorarse en las cuantías correspondientes a los excesos de las contribuciones por haber superado el límite cuantitativo de la reducción de la parte general de la base imponible, durante los ejercicios previos a la percepción de las prestaciones.

$\mathrm{Al}$ igual que ocurría con las prestaciones de los Planes de Pensiones, si la prestación se recibe en forma de renta, se imputará, como rendimientos del trabajo, lo devengado a lo largo del período impositivo; si se opta por la percepción en forma de capital, sobre la totalidad de la prestación se practicará una reducción del 40 
por 100 , siempre que hayan transcurrido más de dos años desde la primera aprer tación para las prestaciones de jubilación y fallecimiento, no siendo exigible el plazo de dos años en el caso de prestaciones por invalidez.

El tratamiento tributario descrito es distinto cuando las aportaciones o cantida des abonadas por contratos de seguro concertados con mutualidades de previsiön social no dan derecho a reducir la parte general de la base imponible como cons secuencia del incumplimiento de los requisitos comentados al principio de este. apartado, es decir, los recogidos en el artículo $46.1 .1 .^{\circ}, 2 .^{\circ}$ y $30^{\circ}$ de la LIRPF. Cuande se produce esta situación las prestaciones por jubilación e invalidez derivadas de dichos contratos se integrarán en la base imponible en la medida en que la cuantat tía percibida exceda de las aportaciones que no hayan podido ser objeto de reduce-t ción o minoración en la base imponible del impuesto por incumplir los anteriores" requisitos, de acuerdo con lo recogido en el artículo 16.2.4. ${ }^{a}$ segundo párrafo de ba LIRPF. Como puede observarse, la cantidad de la prestación a integrar en la basét imponible es distinta, es decir, como hemos visto antes, si las aportaciones sin dani derecho a la reducción de la parte general de la base imponible, por reunir losi requisitos, las prestaciones a que dan derecho se integrarán en su totalidad sinị posibilidad de minorarlas por los excesos de las aportaciones al superar el límite cuantitativo de la reducción; mientras, cuando esas mismas aportaciones no reúnè requisitos para efectuar la minoración de la base imponible, las prestaciones percibidas se integrarán en la cuantía que exceda de las aportaciones que no dieron derecho a la minoración de la parte general de la base imponible. Pues bien, de acuerdo con esta situación descrita, ya sabemos como se deben de integrar las prestaciones por jubilación e invalidez, pero ¿también se califican como rendimientos del trabajo?. Consideramos que no, ya que solamente se califican como rendimientos del trabajo aquellas prestaciones obtenidas por contratos de concertados con mutualidades de previsión social solamente cuando se articulan con unơs requisitos similares a los exigidos a los Planes de Pensiones, por tanto cuando estô no ocurre, como es el caso que comentamos -prestaciones por jubilación e invalidez, cuando las aportaciones no minoran base imponible por incumplimiento de los requisitos exigidos para ello-, nos encontramos con otro rendimiento gravado por el impuesto que se deberá calificar como ganancia patrimonial o rendimiento de capital mobiliario, decantamos por este último. En el caso de prestación pồ causa de fallecimiento tributará por el Impuesto sobre Sucesiones y Donaciones.s.

VI. Contratos De SEguro Colectivo. (ART. 16.2.A).5.' Y ART. 17.2.C) Y D) DE LA LURPRlG:" Con las modificaciones en la redacción de la Disposición Adicional Pximera de 
la Ley 8/1987, operadas por la Ley 30/1995 y 66/1997 se pretende la desaparición de los llamados sistemas de previsión social alternativos por los que se instrumentaban los compromisos por pensiones asumidos por las empresas derivados de obligaciones legales o contractuales entre el empresario y los trabajadores, que podrian revestir distintas fórmulas como fondos internos de la propia empresa, contratos de administración de depósitos... En este sentido dicha Disposición dispone que: en ningún caso resultará admisible la cobertura de tales compromisos mediante la dotación por el empresario de fondos internos. o instrumentos similares, que supongan el mantenimiento por parte de éste de la titularidad de los recursos constituidos", debiendo de estar a lo dispuesto en el Real Decreto 1588/1999, de 15 de octubre, por el que se aprueba el Reglamento sobre instrumentación de los compromisos por pensiones de las empresas con los trabajadores y beneficiarios.

Esto ha obligado a las empresas a instrumentar los compromisos por pensiones mediante un contrato de seguro colectivo $o$, a través de la formalización de un Plan de Pensiones o de ambos. Si el camino elegido hubiera sido el de los Planes de Pensiones nos remitimos a lo dicho anteriormente sobre los mismos. Pero en cambio, si la empresa ha optado por la celebración de un contrato de seguro colectivo para hacer efectivos sus compromisos de pensiones vinculadas a las contingencias de jubilación, invalidez y muerte deben de reunir una serie de requisitos descritos en dicha Disposición Adicional Primera de la Ley 8/1987, que entre otros, señala que: a) debe de revestir la forma de seguro colectivo sobre la vida, en los que la condición de asegurado corresponderá al trabajador y la del beneficiario a las personas en cuyo favor se generen las pensiones según los compromisos asumidos; b) en estos contratos el tomador no podrá ceder ni pignorar la póliza, ni el asegurador conceder anticipos sobre la prestación asegurada al tomador. c) al no existir obligatoriedad de imputar a cada trabajador la prima de este contrato, en aquellos contratos de seguro cuyas primas hayan sido imputadas a los sujetos a los que se vinculen los compromisos por pensiones deberán preverse, de acuerdo con las condiciones pactadas en el compromiso, los derechos económicos de los sujetos en los casos en que se produzca la cesación de la relación laboral previa al acaecimiento de las contingencias cubiertas o se modifique el compromiso por pensiones vinculado a dichos sujetos. d) estos contratos de seguro colectivos se pueden instrumentar entre las mutualidades de previsión social y sus mutualistas en su condición de tomadores del seguro o asegurados o podrán suscribirse con entidades aseguradoras.

Pues bien, si el contrato de seguro colectivo se ha suscrito con una mutualidad de previsión social, el tratamiento fiscal, de las aportaciones como de sus prestaciones, es el mismo que el otorgado para los planes de pensiones, comentado en el anterior apartado, siempre que dichas Mutualidades actúen como instrumentos de previsión social empresarial. 


\section{APORTACIONES}

En el caso que se instrumenten a través de cualquier otra entidad aseguradom el tratamiento dado por el IRPF a las aportaciones efectuadas es el siguiente:

a) en relación con las aportaciones que efectúa el empresario, es decir, las prit mas del contrato satisfechas por el tomador del seguro tendrán la consideración dé gasto deducible de su impuesto sobre la renta si reúne los requisitos del artículọ: 13.3 de la Ley del Impuesto sobre Sociedades.

b) Estas aportaciones o cuotas satisfechas por el empresario cuando sean impu: tadas al trabajador a quien se vincula la prestación. serán para el trabajador un ren dimiento del trabajo en especie, aunque, a diferencia de las aportaciones del promotor al plan de pensiones sistema empleo o a las mutualidades de prevision: social, si se debe efectuar el correspondiente ingreso a cuenta, como parece desprender de la interpretación literal del artículo 95.2 del RIRPF.

Además, el artículo 16.1.e) de la LIRPF, dispone que se efectuará una imputación fiscal que, tendrá carácter obligatoria, en los contratos de seguro de vida quée concedan el derecho de rescate o mediante cualquier otra fórmula permitan su diš posición anticipada por parte de las personas a quienes se vinculen las prestaciónes. Aunque esta imputación fiscal obligatoria no se tendrá que efectuar cuando ël seguro permita la disposición anticipada del derecho de rescate para supuestos de enfermedad grave o desempleo de larga duración.

En aquellos casos en que las primas aportadas por la empresa no se hayan imputado, no existirá gasto deducible para el tomador del seguro ni rendimientos del trabajo para el asegurado, salvo que se conceda el derecho de rescate comen: tado anteriormente, lo que significará, que la imputación, solamente desde el punto fiscal, tendrá el carácter de obligatoria, aunque el derecho no se individualizará hasta que se abonen la correspondiente prestación de jubilación o de invalidez, teniendo en estos casos derecho a una reducción del 40 por 100, si las percibe ế forma de capital, según el artículo 17.2.c) y d) en sus últimos párrafos.

En el caso de haber realizado alguna aportación directa el propio asegurado trabajador al contrato de seguro colectivo, ésta no tendrán la consideración de gasto deducible ni tampoco podrá reducir su base imponible por su importe.

Además, una vez que hemos integrado estas primas como retribuciones edò especie del trabajo, a diferencia de lo que ocurre en los planes y fondos de pensiones 0 con las mutualidades de previsión social, que hemos analizado anteriot:mente, no existe el derecho a reducir la base imponible en el importe de estas ren:tas del trabajo en especie ni por las aportaciones directas que: haya podido efeetuar el asegurado.

Como puede observarse, a través de este mecanismo no produce un difont- 
miento de tributación de la renta destinada a financiar las prestaciones que se obtendrán en el futuro cuando se produzcan las contingencia aseguradas, por tanto, tributan en el ejercicio en que se imputan al trabajador.

\section{Prestaciones}

En cuanto a las prestaciones a percibir de estos contratos de seguro colectivo cuando se produce la contingencia asegurada, y siempre que se haya efectuado la imputación de las primas por parte del empresario a sus trabajadores, el artículo 16.2.a).5. ${ }^{a}$ los califica como rendimientos del trabajo, pero solamente cuando se trate de una prestación por jubilación o invalidez.

Una vez nacido el derecho a percibir la prestación, el asegurado-beneficiario, podrá elegir el recibirla en forma renta, en este caso, solamente tributará como rendimientos del trabajo la parte de la prestación que exceda de las contribuciones imputadas fiscalmente $y$ las aportaciones realizadas por el trabajador, ya que ambas, ya tributaron en su momento. Si la prestación percibida no supera a las aportaciones efectuadas al contrato no habrá que declararla.

En el caso de recibir la prestación en forma capital, habrá que aplicar unos porcentajes de reducción para poder determinar el importe íntegro de estos rendimientos del trabajo, donde la base sobre la que se aplicarán los porcentajes de reducción será la prestación recibida minorada en las contribuciones imputadas fiscalmente y aportaciones directamente realizadas por el trabajador, es decir, el exceso de lo recibido sobre las aportaciones efectuadas.

En relación con la pensión de jubilación, se establece en el artículo 17.2.c) de la LIRPF, una reducción escalona que se aplicará sobre la base definida anteriormente teniendo en cuenta el número de años en que se ha estado satisfaciendo las correspondientes primas:

- los rendimientos que correspondan a primas satisfechas con menos de dos años, se imputarán íntegramente.

- los rendimientos que correspondan a primas satisfechas con más de dos años y menos de cinco de antelación a la fecha en que se perciba la pensión de jubilación, se aplicará una reducción del 40 por 100.

- los rendimientos que correspondan a primas satisfechas con más de cinco años y menos de ocho de antelación a la fecha en que se perciba la pensión de jubilación, se aplicará una reducción del 60 por 100.

- los rendimientos que correspondan a primas satisfechas con más de ocho años de antelación a la fecha en que se perciba la pensión de jubilación, se aplicará una reducción del 70 por 100 . 
Las reducciones anteriores pueden ser sustituidas por una única reducción dế 70 por 100 cuando: a) hayan transcurrido más de doce años desde el pago dét primera prima; b) que el período de permanencia de las primas haya sido superíg; a seis años, siendo el período medio de permanencia de las primas el resultado de calcular el sumatorio de las primas multiplicadas por su número de años de perte manencia y dividirlo entre la suma total de las primas satisfechas, artículo 11.2 dis RIRPF; c) que el contrato de seguro haya sido concertado a partir del 31 de dicieme bre de 1994, según la Disposición Transitoria séptima de la LIRPF, lo que signifíca. que esta reducción se podrá a aplicar a aquellas pensiones de jubilación obtenidaśt a partir del año 2.007 .

A la hora de determinar la parte de rendimiento total obtenido que correspong de a cada prima cuando los contratos de seguro de vida tengan primas periódicít o extraordinarias habrá que seguir el procedimiento establecido en el artículo 12:4 del RIRPF, es decir, multiplicar el rendimiento total por el cociente obtenido dè resultado de multiplicar la prima correspondiente por el número de años desde quét se pago hasta el cobre de la pensión / sumario de los productos resultantes de nuile tiplicar cada prima por el número de años transcurridos desde que fue satisfecthâ hasta el cobreo de la percepción.

Teniendo la obligación las entidades aseguradoras de desglosar la parte de rendimiento que corresponda a cada una las primas pagadas, artículo 11.5 del RIRPR

Cuando se trate de la prestación por invalidez las reducciones serán la siguien-: tes, artículo 17.2.d) de la LIRPF:

- tratándose de indemnizaciones por invalidez absoluta y permanente para todb: trabajo y por gran invalidez en los mismos términos establecidos por la normativa reguladora de los planes y fondos de pensiones, le corresponde una reducción del 60 por 100 , artículo 11.3 del RIRPF. No siendo de aplicación la exención del artés culo 7 de dicha Ley.

- en el caso de no reunir los requisitos anteriores, los rendimientos derivades de otras prestaciones por invalidez, tendrán una reducción será del $\mathbf{4 0}$ por 100.

- si la prestación de invalidez deriva de contratos de seguro concertados desde el 31 de diciembre de 1994, que tengan más de doce años de antigüiedad y que ê: período de permanencia de las primas satisfechas a lo largo del contrato haya sidố superior a seis años, tendrán derecho a una reducción del 70 por 100.

En caso de fallecimiento del asegurado la prestación la recibirá otra persona, el beneficiario, y en este caso, la prestación recibida no tributará por el IRPF, sino pot el Impuesto sobre Sucesiones y Donaciones, con independencia de que la misina la reciba en forma de renta o en forma de capital, comø consecuencta de la nueva redacción del artículo 3.1c) de la Ley del Impuesto sobre Sucesiones y Donacionents dada por la Disposición Final Primera de la LIRPF, con el siguiente tenor: alc) Iapere: 
cepción de seguros sobre la vida, cuando el contratante sea persona distinta del beneficiario, salvo los supuestos expresamente regulados en el articulo 16.2.a) de la Ley del Impuesto sobre la Renta de las Personas Fisicas".

\section{Bibliografía}

- AA.VV. El Impuesto sobre la Renta de las Personas Físicas (Ley 40/1998). Ed. Comares. 1999 AA.VV. (Dirigidos por G. Oron Moratal): Los Nuevos Impuestos sobre la Renta de las Personas Físicas y sobre la Renta de no Residentes. (Comentarios a las leyes 40/1998 y 41/1998, con sus respectivos Reglamentos aprobados por Reales Decretos 214/1999, de 5 de febrero y 326/1999, de 26 de febrero). Madrid. 1999.

AA.VV. (Dirigidos por M. Cruz Amorós): Comentarios a la Leyes Tributarias: T. I. Vol. $1^{\circ}$ y 20: Impuesto sobre la Renta de las Personas Físicas. Edt. Edersa. Madrid. 1998.

- AA.VV.: El Nuevo Impuesto sobre la Renta de las Personas Físicas. Edt. Lex Nova. Valladolid. 1999.

- CAZORLA PRIETO, Luis María y PEÑa ALONSO, José Luis: El Impuesto sobre la Renta de las Personas Físicas Ley 40/1998 y su Reglamento. Edt. Aranzadi, 1999.

CORDÓN EZQUERRO, T.; MANCHE $\backslash O$ GARCÍA-LAJARA, S.; MOLINA FERNÁNDEZ, J.: Impuesto sobre la Renta 1999. Comentarios y casos prácticos. Edita Centro de Estudios Financieros. Madrid. 1999.

- CORREAS GONZÁlEZ, L.M.; GARCÍA MONTENEGRO, L.; HOYO ORTIGOSA, C. del; LINARES SÁEZ, M. D. LÓPEZ CARBAJO, J.M.; PÉREZ-CAMPANERO FERNÁNDEZ, J.L.; SANTA CRUZ DEL HOYO, M.L.: Guía del la Ley del Impuesto sobre la Renta. Ley 40/1998, de 9 de diciembre, del Impuesto sobre la Renta de las Personas Físicas. Edt. CISS. $1^{\mathbf{a}}$. edic. Bilbao. 1999.

ENRIQUE FERNÁNDEZ HERRERO; JUAN IGNACIO GOROSPE OVIEDO: Reflexiones en torno al Nuevo Impuesto sobre la Renta de las Personas Físicas. Edt. Civitas. 1999

- PÉREZ ROYO, Ignacio. Manual del Impuesto sobre la Renta de las Personas Físicas. (Análisis de la nueva Ley y de su Reglamento). Edt. Marcial Pons. Madrid. 1999.

SEGARRA, Ángel y FIGAROLAS, Joan de: El Nuevo Impuesto sobre la Renta de las Personas Físicas. (Análisis y comentarios). Edt. Deusto.1999. 